\title{
GEORG ECKERT UND DAS INTERNATIONALE SCHULBUCHINSTITUT IN BRAUNSCHWEIG
}

Georg Eckert (1912-1974) einen Platz als Akteur in der Geschichte der Geschichtswissenschaft zuzuweisen, bedarf der Erklärung. Gilt er doch eher als Pädagoge ${ }^{1}$, dessen Name landläufig mit den Schulbüchern in Verbindung gebracht wird durch das nach ihm benannte Georg-Eckert-Institut für internationale Schulbuchforschung in Braunschweig (GEI). Einer breiteren Öffentlichkeit ist das Institut durch die maßgeblich von Eckert Anfang der 1970er Jahre initiierten deutsch-polnischen Schulbuchkonferenzen in Geschichte und Geographie bekannt geworden; ihr Ergebnis, eine Reihe »Gemeinsamer Empfehlungen für Schulen und Verlage «, hat in den 1970er und 1980er Jahren die bundesdeutsche und internationale Öffentlichkeit nachhaltig beschäftigt und ist überaus kontrovers diskutiert worden ${ }^{2}$. Der übrigens nicht nur in der Bundesrepublik, sondern auch in der damaligen Volksrepublik Polen umstrittene Charakter der Gemeinsamen Empfehlungen hat dazu geführt, daß das Institut von seinen Gegnern bisweilen in polemischer Absicht als rdeutschpolnisches Institut bezeichnet worden ist. Der durch diese polemische $\mathrm{Zu}$ spitzung erzeugte Ruf verschleierte mehr als er erklärte; er verengte den Blick auf die tatsächliche Bandbreite und Eigentümlichkeit der Aktivitäten, die vom GEI entweder ausgingen oder in ihm zusammenliefen und weitergegeben wurden ${ }^{3}$.

Als »Professor für Pädagogik « bezeichnet ihn Matthias BODE, Auswärtige Kulturpolitik zwischen Demokratisierung und Internationalisierung. Die frühe Bundesrepublik als Partner der UNESCO (Magisterarbeit), Göttingen 2005, S. 6.

2 Empfehlungen für Schulbücher für Geschichte und Geographie in der Bundesrepublik Deutschland und in der Volksrepublik Polen, hg. von der Gemeinsamen deutschpolnischen Schulbuchkommission, Braunschweig 1977, mit zahlreichen Nachdrucken an unterschiedlichen Druckorten; Die deutsch-polnischen Schulbuchempfehlungen in der öffentlichen Diskussion der Bundesrepublik Deutschland. Eine Dokumentation eingeleitet von Wolfgang JACOBMEYER, Braunschweig 1979; ein Versuch einer Gesamtwürdigung: Thomas STROBEL, Die Gemeinsame deutsch-polnische Schulbuchkommission. Ein spezifischer Beitrag zur Ost-West-Verständigung 1972-1989, in: Archiv für Sozialgeschichte 45 (2005), S. 253-268.

3 Vgl. Internationale Verständigung. 25 Jahre Georg-Eckert-Institut fuir internationale Schulbuchforschung in Braunschweig, hg. von Ursula A.J. BECHER und Rainer RIEMENSCHNEIDER unter Mitwirkung von Roderich HENRŸ, Hannover 2000; für die aktuelle Arbeit: Eckert. Das Bulletin 01 (Sommer 2007), Redaktion: Verena Radkau. 
Auch unsere Fachwelt hat ein eher diffuses Bild von der Person und dem Wirken Eckerts. Dies mag daran liegen, daß Eckert auch auf Gebieten aktiv geworden war, die weit über das Tätigkeitsfeld eines Hochschullehrers hinausgingen. So leitete er das Internationale Schulbuchinstitut (ISBI) an der KantHochschule in Braunschweig, das er 1951 begründet hatte. 1949 gehörte er zu den Gründungsvätern des Verbandes der Geschichtslehrer Deutschlands und war sein langjähriger Schatzmeister (1949-1964). Er gründete das Institut für Sozialgeschichte Braunschweig/Bonn und war außerdem Begründer und seit 1961 Schriftleiter des »Archivs für Sozialgeschichte«. Im Vorstand der Friedrich Ebert-Stiftung war er zugleich aktives Mitglied der SPD und der Gewerkschaft für Erziehung und Wissenschaft (GEW) sowie Vorsitzender des Kuratoriums Unteilbares Deutschland in Braunschweig. Gleichzeitig gehörte er zu den frühesten Mitgliedern der Deutschen UNESCO-Kommission (seit 1950) und war von 1964 bis 1974 deren Vorsitzender ${ }^{4}$. Auf allen diesen Feldern hat er sich mit einer immensen Arbeitsleistung engagiert; davon legt sein umfangreicher Nachlaß Zeugnis ab, und Zeitgenossen, die ihn näher gekannt haben, bestätigen dies. Eckert war nicht nur ein begeisterter und begeisternder Lehrer, der seine Hörer stark und nachhaltig beeindruckte ${ }^{5}$; er wußte sich auch geschickt der Medien zu bedienen und verstand es, komplizierte Sachverhalte in leicht verständlicher Form einer breiten Öffentlichkeit zu vermitteln. So erschien er bald als Hochschullehrer, bald als Partei- oder Verbandspolitiker und schließlich als Kulturpolitiker. Daß Georg Eckert auch ein international anerkannter Forscher der Geschichte der deutschen Arbeiterbewegung war, ist dabei etwas in Vergessenheit geraten ${ }^{6}$. Eine zusätzliche Schwierigkeit, ihn seiner Bedeutung entsprechend wahrzunehmen, mag darin begründet sein, daß er in der Fachhistorikerschaft ein "Außenseiter« war? Ein Außenseiter allerdings mit ausgeprägtem Kommunikationsvermögen und starker persönlicher Ausstrahlungskraft, die es ihm erlaubten, an der Wiederanknüpfung des transnationalen Dialogs nach dem Zweiten Weltkrieg wirkungsvoll mitzuwirken. Diese beiden Aspekte der Persönlichkeit Eckerts sollen Gegenstand der folgenden Ausführungen sein.

Georg Eckerts Wirken in der UNESCO wird speziell von BODE, Auswärtige Kulturpolitik (wie Anm. 1) untersucht.

5 Hierza der bewegende Bericht von einem seiner früheren Schüler: Heinz WARMBOLD, Sprechen möchte ich von Georg Eckert, dem Lehrer, in: In memoriam Georg Eckert (1912-1974), Braunschweig 1974, S. 13-15.

6 Vgl. u.a. Georg ECKERT (Hg.), 1863-1963. Hundert Jahre deutsche Sozialdemokratie. Bilder und Dokumente, Hannover 1963; DERS., Liebknecht, Wilhelm: Briefwechsel mit deutschen Sozialdemokraten, Bd. 1: 1862-1878, Frankfurt a.M. 1973.

7 Emst HINRICHS, Falk PINGEL, Georg Eckert (1912-1974) und die internationale Schulbuchforschung, in: Paul LEIDINGER (Hg.), Geschichtsunterricht und Geschichtsdidaktik vom Kaiserreich bis zur Gegenwart. Festschrift des Verbandes der Geschichtslehrer Deutschlands zum 75jährigen Bestehen, Stuttgart 1988, S. 334-349, hier S. 334. 


\section{Als Außenseiter in der Historikerzunft}

Eckerts Außenseiterposition in der deutschen Historikerschaft hat mehrere Ursachen. Es lassen sich mindestens drei Elemente erkennen: sein ungewöhnliches oder auch untypisches Ausbildungs- und Berufsprofil, sein politisches Engagement als Sozialist bzw. Sozialdemokrat und als Gegner des Naziregimes sowie seine damit zusammenhängende Konzeption von Forschung und Lehre bzw. Lehrerausbildung.

\section{Sein atypisches Ausbildungs- und Berufsprofil}

Georg Eckerts Vita hat durch seine familiäre Sozialisation ihre entscheidende Richtung erfahren ${ }^{8} .1912$ in Berlin geboren, entstammte er einem sozialistisch geprägten Elternhaus. Seine Mutter Louise Eckert-Ryschawy hatte an der Russischen Revolution von 1905 teilgenommen, sein Vater war Ingenieur und als Chefredakteur einer technischen Zeitschrift tätig; als engagierter Sozialdemokrat konnte er nicht mit einer leitenden Stellung als Ingenieur in der Wirtschaft rechnen. Nach dem Abitur 1931 immatrikulierte sich Eckert an der Berliner Universität in den Fächern Geschichte, Geographie, Germanistik sowie in der Volks- und Völkerkunde. Zu den ihn prägenden Lehrern zählte Eckert den Lassalle-Biographen Hermann Oncken, den Althistoriker und Soziologen Arthur Rosenberg, der ihn nach Eckerts Worten "sachkundig in die Welt des Marxismus einführte«, und den Reformpädagogen Fritz Karsen. Neben seinem eifrig betriebenen Studium engagierte sich Eckert in den sich zuspitzenden Krisenjahren in der Politik. Schon als Schüler war er der Sozialistischen Arbeiterjugend und der SPD beigetreten, im März 1932 übernahm er den Vorsitz im Sozialistischen Studentenbund an der Universität Berlin und betätigte sich auch im Reichsbanner Schwarz-Rot-Gold. Da er sich politisch exponiert hatte, befürchtete er nach dem Machtantritt Hitlers eine Verhaftung, exmatrikulierte sich in Berlin und tauchte zeitweilig in Hildesheim unter, wo er als wissenschaftlicher Hilfsarbeiter am Pelizaeusmuseum unterkam. Im Sommersemester 1933 setzte er das Studium in Bonn fort, diesmal mit dem Schwerpunkt Völkerkunde, da er dieses Fach für politisch weniger exponiert hielt als die Geschichte. Schon im November 1935 wurde er mit einer durch Hermann Trimborn betreuten Dissertation zum Thema »Der Einfluß des Geschlechtsund Familienlebens auf die Bevölkerungsbewegung mikronesischer Inseln«

Vgl. zum Folgenden vor allem: Hans-Peter HARSTICK, Georg Eckert (1912-1974). Wegbereiter einer neuen Konzeption von Geschichte in Wissenschaft und Unterricht, in: Internationale Verständigung (wie Anm. 3), S. 105-115, mit einer wichtigen Anmerkung zu Quellengrundlage und Forschungsstand; vgl. auch Rosemarie RÜMENAPF-SIEVERS, Georg Eckert und die Anfänge des Internationalen Schulbuchinstituts, in: Ibid. S. 116-122. 
promoviert. Ein halbes Jahr später, im Juni 1936, legte er ebenfalls in Bonn die wissenschaftliche Prüfung für das Lehramt an Höheren Schulen in den Hauptfächern Geschichte und Erdkunde und in den Nebenfächern Deutsch und Deutsche Volkskunde ab; im September 1938 folgte die Pädagogische Prüfung, diesmal vor dem Landesprüfungsamt Berlin. Bis zum Kriegsausbruch war er als Assessor an mehreren Bildungsanstalten um und in Berlin tätig; im September 1943 sollte dann die Ernennung zum Studienrat erfolgen. Am Frankreichfeldzug nahm Eckert als Funker bei der Infanterie teil, und von 1941 bis 1944 wurde er als Meteorologe an die Marine-Wetterwarte in Saloniki abkommandiert. Dort hatte er als Dienststellenleiter Zeit für volkskundliche bzw. ethnographische Feldstudien über Griechisch-Mazedonien, die er in einer Reihe von Aufsätzen veröffentlichte. Im Februar 1943 habilitierte er sich während eines Fronturlaubs in Bonn mit einer Arbeit über die Chibchahochkultur in Westkolumbien, die dann 1948 mit dem Titel »Totenkult und Lebensglaube im Caucatal« als erster Band einer von Trimborn und Eckert begründeten Reihe »Kulturgeschichtliche Forschungen « in Braunschweig bei Limbach erschien. Die Probevorlesung hatte als Thema »Die Kopfjagd bei den südamerikanischen Hochkulturvölkern «, und die Lehrprobe handelte von der "Reichspolitik der Inka«. Damit hatte Eckert, der für diese Arbeiten Spanisch gelernt hatte, die Lehrbefugnis für Amerikanistik erworben. Ausgeübt hatte er diese Facultas jedoch nie, denn die weitere Entwicklung des Krieges führte ihn in eine andere Richtung.

\section{Sein politisches Engagement: im Widerstand gegen den Nationalsozialismus}

Hatte sein politisches Engagement als Sozialist ihn bereits wegen befürchteter Repression von seinem Hauptfach, der Geschichte, abgebracht, so führte seine oppositionelle Einstellung zum NS-Regime ihn in den Widerstand. Nach dem 20. Juli 1944 nahm Eckert mit der Griechischen Befreiungsfront EAM und ihrer militärischen Kampforganisation, der Griechischen Volksbefreiungsarmee ELAS, Verbindung auf. Er gründete das Komitee Freies Deutschland für Mazedonien und unterstellte sich mit mehreren Tausend zurückgebliebener deutscher Soldaten der ELAS, nachdem am 31. Oktober 1944 der Wehrmachtsbefehl ergangen war, daß die deutschen Truppen Saloniki, den Sitz des Oberbefehlshabers der Heeresgruppe $\mathrm{E}$, zu räumen hatten. In britischer Gefangenschaft zunächst in Italien zog sich Eckert ein schweres Lungenleiden zu, so daß er im August 1945 mit einem Lazarettzug nach Goslar gebracht wurde. Hier machte er die Bekanntschaft des damaligen Braunschweigischen Ministerpräsidenten Alfred Kubel, der selbst auch im Widerstand tätig gewesen war und der Eckert eine Dozentur für Geschichte an der Hochschule für Lehrerbildung in Braunschweig anbot. Eckert nahm das Angebot an und trat 
am 1. November 1946, mit 34 Jahren, die Stelle an der inzwischen in »KantHochschule« umbenannten Institution an. So kam es, daß ein habilitierter Ethnologe im Bereich der Amerikanistik dann im April 1948 die Ernennung zum Professor für Geschichte und Methodik des Geschichtsunterrichts erhielt. Wahrlich ein nicht alltägliches Ereignis.

Es war weniger die politische Haltung, die Eckert die Tätigkeit an einer Hochschule eintrug, als vielmehr die neue Bildungspolitik der Nachkriegszeit, die auf eine stärkere Verwissenschaftlichung der Ausbildung an den Pädagogischen Hochschulen abzielte. Eckert besaß dieses wissenschaftliche Profil, wenngleich er es auf dem Gebiet der Völkerkunde erworben hatte. Geschichte war in der ersten Phase seines Studiums das Hauptfach gewesen, und er hatte auch in diesem Fach praktische Lehrerfahrungen sammeln können, wenn auch nicht in der Volksschule, sondern an höheren Bildungsanstalten. Immerhin brachte Eckert damit wichtige Voraussetzungen für eine wirkungsvolle Lehrtätigkeit im Sinne der neuen Bildungspolitik in die Hochschule ein.

Als schwieriger erwies sich indes der Umstand, dem Widerstand gegen den Nationalsozialismus angehört zu haben. Eckert machte die Erfahrung einer asymmetrischen Geltung der Vertreter des deutschen Widerstandes: Im Innern wurden sie, zumal wenn sie im Ausland tätig gewesen waren, von weiten Bevölkerungskreisen mit Mißtrauen betrachtet und beargwöhnt. Willy Brandt ist hierfür das wohl prominenteste Beispiel. Widerstandskämpfer polarisierten die Meinung der Deutschen nach 1945. Im Ausland jedoch wurden sie als die Vertreter des »besseren«, des »anderen« Deutschlands weitgehend geschätzt. Aufgrund dieser Vergangenheit genoß Eckert von vornherein das Vertrauen der Besatzungsmacht, bei der er sich Anerkennung verschafft hatte. Von der britischen Militärregierung wurde er als Gutachter für Schulbuchfragen herangezogen, was ihm in konservativen Kreisen auch nicht honoriert wurde, stand die Reeducation-Politik in diesen Kreisen doch im Verdacht, eine Komplementärstrategie zur Entnazifizierung ${ }^{9}$ zu sein, so daß deren Befürworter vielfach als Erfüllungsgehilfen des Auslands disqualifiziert wurden. Eckerts $\mathrm{Zu}$ sammenarbeit mit den britischen Erziehungsbehörden geschah nicht aus Opportunismus, sondern aus Überzeugung und aus seiner Erfahrung als Gegner des Nationalsozialismus. Dabei war Eckert kein politischer Eiferer, sondern eher tolerant: Er stand uneingeschränkt hinter der seit der Wiedereröffnung der Hochschule am 12. November 1945 bei der britischen Militärregierung durchgesetzten, einmalig in Deutschland praktizierten Rekrutierungspolitik, die darin bestand, zur Immatrikulation an der Kant-Hochschule auch frühere $\mathrm{HJ}$-Führer und BDM-Führerinnen zuzulassen ${ }^{10}$. Weltkriegs bis zur Gründung der beiden deutschen Staaten 1914-1949, München 2003, S. $959 f f$. 


\section{Seine Forschungskonzeption: Sozial- und Wirtschaftsgeschichte als Imperativ einer neuen Geschichtsdidaktik}

Mit starkem pädagogischem Ethos nahm Eckert seine Arbeit als Hochschullehrer auf. Es war die Begeisterung für die Lehre und das Verantwortungsgefühl gegenüber sseinen Studenten, die ihn in kritischen Momenten davon abhielt, die Hochschule zu verlassen und in die praktische Politik überzuwechseln ${ }^{11}$. Bezeichnenderweise ist er während einer gegen ärztlichen Rat gehaltenen Vorlesung vor seinen Studenten im Alter von 63 Jahren an einem Herzinfarkt gestorben.

Mit seiner oppositionellen Haltung gegenüber dem Nationalsozialismus legitimierte Eckert die demokratische Fundierung seiner Lehrtätigkeit. Dies kommt in seinem am 1. November 1945 abgefaßten Lebenslauf »mit Blick auf Wiedereinstellung in den Schuldienst« zum Ausdruck:

Ich erkläre abschließend an Eidesstatt, daß ich bis 1933 offen und von da ab geheim pausenlos und mit allen mir zur Verfügung stehenden Mitteln an der Befreiung meiner Heimat vom Nationalsozialismus gearbeitet habe. Ich bitte mir jetzt Gelegenheit zu geben, meinen Anteil an der Erziehung eines jungen demokratischen Deutschlands zu leisten ${ }^{12}$.

Wenn die leicht emphatisch wirkende Formulierung sich auch aus den besonderen Zeitumständen erklärt, so wird in dem Text doch das demokratische Selbstverständnis deutlich, das zu artikulieren unter der Diktatur lebensgefährlich gewesen war. Die existentielle Erfahrung, daß Demokratie und Freiheit etwas Fragiles sind und gegen Gefährdungen errungen und behauptet werden müssen, hat Eckert stets begleitet und bildete den Grundton seines Denkens. Sie stand am Anfang seiner Bestrebungen, nach dem Krieg Netzwerke der Zivilgesellschaft aufzubauen oder in ihnen aktiv mitzuwirken. Als Beispiel sei hier der von ihm begründete Geschichtspädagogische Arbeitskreis genannt, der auch Einfluß auf die Lehrplangestaltung in der britischen Zone gewinnen sollte $^{13}$. In ihm sind Eckerts "Grundthesen zur Reform des Geschichtsunterrichts « vom Februar $1948^{14}$ entstanden, die der wenig später gegründeten GEW als Richtschnur dienten. Die wichtigsten Punkte dieser Thesen seien hier kurz wiedergegeben:

In der neuen Schule des demokratischen Deutschlands wird Geschichte als Voraussetzung für eine wirkungsvolle staatsbürgerliche Erziehung zu den wichtigsten Unterrichtsfächern gehören [...]. Der Geschichtsunterricht muß daher gründliche Kenntnisse geschichtlicher Tatsachen und Zusammenhänge vermitteln und zu selbständigem geschichtlichen Denken, als der Voraussetzung zu eigener politischer Urteilsbildung erziehen.

11 Hierzu HinRICHS, PINGEL, Georg Eckert (wie Anm. 7), S. 338.

12 Zitiert bei HARSTICK, Georg Eckert (wie Anm. 8), S. 107.

13 Ulrich MAYER, Neue Wege im Geschichtsunterricht? Studien zur Entwicklung der Geschichtsdidaktik und des Geschichtsunterrichts in den westlichen Besatzungszonen und in der Bundesrepublik Deutschland 1945-1953, Köln, Wien 1986, S. $183 \mathrm{ff}$.

14 HARSTICK, Georg Eckert (wie Anm. 8), S. 109. 
Um das im Dritten Reich erschütterte Vertrauen zur Geschichte wiederherzustellen, ist im Geschichtsunterricht auf strengste wissenschaftliche Gewissenhaftigkeit und weltanschauliche Toleranz zu achten. Wissenschaftliche Objektivität ist jedoch nicht mit Standpunktlosigkeit des Erziehers zu verwechseln.

Neben der politischen Geschichte steht die Volks- und Kulturgeschichte im Mittelpunkt des neuen Geschichtsunterrichts [...]. Es ist dabei nicht nur die Kultur der Oberschichten, sondern vor allem auch die der breiten Volksmassen, ihre Wirtschaft, ihr Denken, ihr tägliches Leben, ihr politisches und gesellschaftliches Streben zu behandeln. Besonderes Gewicht ist auf die mannigfaltigen Triebkräfte des geschichtlichen Geschehens zu legen: die Bedeutung geistiger und ökonomischer Faktoren, den Einfluß großer Persönlichkeiten oder die Auswirkung gruppen- und massenpsychologischer Erscheinungen. Stärkere Beachtung ist den in der Vergangenheit vernachlässigten wirtschaftlich-gesellschaftlichen Faktoren zu widmen.

Die deutsche Geschichte muß stärker in den Zusammenhang des »Abendlandes« und in die Weltgeschichte eingebettet werden, mwenn nicht auch in Zukunft mangelnde Kenntnis der Welt und fehlendes Verständnis fuir andere Völker und Kulturen zu Fehlentwicklungen und zu engherzigem Chauvinismus führen sollen«.

Der Geschichtsunterricht hat nicht zuletzt der Friedensidee zu dienen ${ }^{15}$.

Lassen wir die geschichtsdidaktischen Aussagen außer acht, die Eckert im Urteil der modernen Forschung als einen der wanregendsten Geschichtsdidaktiker

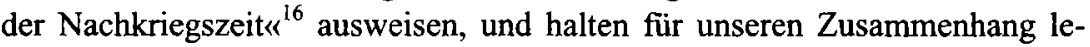
diglich die Konsequenzen fest, die sich aus diesen Postulaten für die Geschichtswissenschaft als Bezugswissenschaft ergeben. Hier steht an erster Stelle die Betonung der Wissenschaftlichkeit der Lehrerausbildung, die sich wie ein roter Faden durch die Überlegungen zieht ${ }^{17}$. Doch an welcher wissenschaftlichen Richtung sollte sich die Lehrerbildung orientieren? Gefordert wird neben der traditionellen politischen Geschichte und der Betrachtung groBer Persönlichkeiten nun auch eine Sozial- und Wirtschaftsgeschichte, die das Augenmerk auf die gesamte Gesellschaft richtet und die imstande ist, geschichtliche Entwicklungen nicht nur nachzuerzählen, sondern sie auch hinsichtlich ihrer komplexen Bedingungsfaktoren zu erklären. Hier drückt sich ein Geschichtsbewußtsein aus, das, ohne sich ganz von der traditionellen geisteswissenschaftlich bestimmten Geschichte etwa eines Erich Weniger zu trennen, doch eine an materialistischer Aufklärungshistorie inspirierte Kom-

15 Zitiert nach: Georg ECKERT, Grundprobleme des Geschichtsunterrichts (Der Beitrag der Gewerkschaft Erziehung und Wissenschaft zur Reform des Geschichtsunterrichts), in: Geschichtsunterricht in unserer Zeit. Grundfragen und Methoden, Braunschweig 1952, S. $147 \mathrm{ff}$.

16 Ulrich MEYER, zit. bei HARSTICK, Georg Eckert (wie Anm. 8), S. 109.

17 Ähnlich auch die Formulierung "Wissenschaftlichkeit des Geschichtsunterrichts«, Georg ECKERT, Geschichtsunterricht und Völkerverständigung. Zum Problem der internationalen Schulbuchverbesserung, in: Geschichtsunterricht in unserer Zeit (wie Anm. 15), S. 140-146, hier S. 146. 
ponente aufnahm ${ }^{18}$. Geschichtsunterricht, als im Dienste staatsbürgerlicher Erziehung stehend, sollte in erster Linie aufklärend wirken.

Diese Thesen waren nicht nur innovativ für die Geschichtsdidaktik, die in der Nachkriegszeit weiterhin der geisteswissenschaftlichen Pädagogik verpflichtet blieb ${ }^{19}$. Ihre Umsetzung in Lehre und Unterricht erforderte auch ganz neue Lehrmaterialien, die es aber zu jener Zeit nicht gab. Um diesen Mangel wenigstens etwas zu lindern, wurden die »Beiträge zum Geschichtsunterricht« geschaffen, eine 36 Lieferungen umfassende Sammlung von Quellen- und Arbeitsheften zu bestimmten Themen der Geschichte, die Eckert im Auftrag eines Geschichtspädagogischen Arbeitskreises besorgte. Die Liste der Titel gibt Aufschluß über die Themen, die in den Nachkriegsjahren als besondere Desiderate empfunden worden sind ${ }^{20}$. Auch hier ist der Anteil Eckerts beträchtlich. Diese Materialien waren ein Notbehelf für den Geschichtsunterricht, um die größten Lücken zu füllen. Sie führten aber auch ständig vor Augen, daß die Bezugswissenschaft keine oder doch nur sehr unzureichende Referenzwerke für den neuen Unterricht zur Verfügung stellen konnte. So ist von diesem Neuansatz des Geschichtsunterrichts auch aus rein praktischen Gründen die programmatische Forderung nach Innovation in der Geschichtsforschung ausgegangen, und man geht sicher nicht fehl in der Annahme, daß die in den 1960er Jahren allmählich sich herausbildende historische Sozialwissenschaft innerhalb der deutschen Geschichtswissenschaft auch von den pragmatischen Impulsen aus der Geschichtsdidaktik heraus begünstigt wurde ${ }^{21}$.

Eckert war an dieser Entwicklung nicht nur aufgrund seiner didaktischen Bemühungen beteiligt. Er hat sie auch teils durch eigene Forschungen, teils durch Forschungsorganisation beeinflußt. Aus seiner eigenen Forschung zur Geschichte der deutschen Arbeiterbewegung seien hier folgende Veröffentlichungen genannt: Wilhelm Bracke und die Anfänge der deutschen Arbeiterbewegung (Braunschweig 1957); Die Braunschweiger Arbeiterbewegung un-

Die Tradition der Aufklärung im Denken Eckerts unterstreicht Rolf WERNSTEDT, Die Gründung des Georg-Eckert-Instituts aus politischer und parlamentarischer Sicht, in: Internationale Verständigung (wie Anm. 3), S. 124-128, hier S. 125.

19 Hierzu HINRICHS, PINGEL, Georg Eckert (wie Anm. 7), S. 337; vgl. Jürgen MIROw, Geschichte des deutschen Volkes von den Anfängen bis zur Gegenwart, Köln 1996, S. 1028. Eine Liste der »Beiträge zum Geschichtsunterricht« befindet sich im Anhang.

${ }^{21}$ Der hier geschilderte Vorgang einer Vorreiterrolle der Didaktik in Bezug auf die Geschichtswissenschaft war kein Einzelfall. Rainer Bendick hat mich darauf hingewiesen, daß Karl-Ernst Jeismanns Konzept des "Geschichtsbewußtseins« (1980) nicht nur die Entwicklung der westdeutschen Geschichtsdidaktik stark geprägt hat, sondem auch wichtige Impulse für die Forschung im Bereich der »Geschichtskultur« und der »Erinnerungskultur« gegeben hat. Siehe hierzu die Artikel »Geschichtskultur« von Jörn RÜSEN und »Geschichtsbewußtsein« von Karl-Ernst JEISMANN, beide in: Klaus BERGMANN u.a. (Hg.), Handbuch der Geschichtsdidaktik, Seelze-Velber ${ }^{5} 1997$, S. 38-44, mit weiterer Literatur. Es handelt sich hier um einen Aspekt in der Geschichte unseres Faches, der eine genauere Untersuchung verdiente. 
ter dem Sozialistengesetz (Braunschweig 1961); 100 Jahre Braunschweiger Sozialdemokratie, Teil 1: Von den Anfängen bis zum Jahre 1890 (Hannover 1965). Hinzu kamen mehrere Editionen, wie der Briefwechsel Wilhelms Liebknechts mit Marx und Engels (Den Haag 1963) und der Briefwechsel Wilhelm Liebknechts mit deutschen Sozialdemokraten (Assen 1973) - eine Edition, die in der Forschung als vorbildlich gilt ${ }^{22}$. Die Erforschung der Arbeiterbewegung war in der deutschen Geschichtswissenschaft der Nachkriegszeit marginal, sie war eine Domäne der in der DDR betriebenen Historie ${ }^{23}$. Eine zusätzliche Schwierigkeit für Eckert, sich in Fachkreisen Gehör zu verschaffen, bestand darin, daß es zwischen Geschichtsdidaktikern und Geschichtswissenschaftlem gleichsam strukturell bedingte Verständigungsprobleme und "Spannungen« gab, die teilweise bis in die Gegenwart hineinwirken:

Bis heute [d.h. 1990; R.R.] verstehen sich Geschichtsdidaktik und Geschichtswissenschaft nicht als gleichberechtigte Partner in derselben Disziplin; die meisten Fachwissenschaftler sehen in der Didaktik nur die Methode, das, was sie erarbeitet haben, dem Verständnis eines breiteren Publikums zugänglich zu machen. Die Wissenschaft erforscht, die Didaktik simplifiziert ${ }^{24}$.

Unter diesen Bedingungen hatte es Eckert als Vertreter einer pädagogischen Hochschule nicht leicht, als vollwertiger Fachwissenschaftler in der deutschen Historikerschaft wahrgenommen zu werden. Ganz anders im Ausland, im westlichen zumal, wo Eckert als einer der wenigen westdeutschen Historiker die Geschichte der Arbeiterbewegung auf internationalen Kolloquien vertrat und hohe Anerkennung genoß. In der Organisation von Forschung war Eckert auf längere Sicht erfolgreicher. Stellvertretend sei hier lediglich das »Archiv für Sozialgeschichte« genannt, dessen Programmatik überraschende Parallelen zu den Zielsetzungen der Zeitschrift "Geschichte und Gesellschaft « aufweist ${ }^{25}$. Dies alles zusammengenommen - die geschichtsdidaktische Position, die Erforschung der Arbeiterbewegung und die Organisation von Forschung - läßt Eckert als einen "Wegbereiter einer neuen Konzeption von Geschichte in Wissenschaft und Unterricht« (so der Titel des Artikels von Harstick) erscheinen.

Eine Bibliographie der Schriften Eckerts im Archiv für Sozialgeschichte Band XIV/1974, S. XIVff.

${ }^{23}$ Falk PINGEL, Geschichte unserer Zeit - Zeit für Geschichte? Geschichtsdidaktik und Geschichtswissenschaft in ihrem Verhältnis zur Zeitgeschichte in den Westzonen und in der Bundesrepublik, in: Tel Aviver Jahrbuch für deutsche Geschichte XIX (1990), S. 233258, hier S. 249.

24 Ibid. S. 233.

$25 \mathrm{Vgl}$. hierzu die Gegenüberstellung der beiden Editorials im ersten Band des "Archiv für Sozialgeschichte« 1961, und im ersten Heft von »Geschichte und Gesellschaft«, 1975, bei HARSTICK, Georg Eckert (wie Anm. 8), S. 105. 
Die internationale Schulbuchrevision

Ein Beitrag zur Völkerverständigung und zur Rückkehr in die Ökumene

\section{Gründerjahre}

Eckert hat, den Hiatus zwischen Geschichtswissenschaft und Geschichtsdidaktik bzw. -unterricht ignorierend, beide Disziplinen in der Schulbuchrevision zusammengeführt. Kennzeichnend hierfür war die Gründung des Internationalen Instituts für Schulbuchverbesserung an Ostern $1951 \mathrm{im}$ Rahmen einer Historiker- und Geschichtslehrertagung aus neun Staaten (Dänemark, England, Frankreich, den Niederlanden, Norwegen, Schweden, der Schweiz, den USA und der Bundesrepublik) an der Kant-Hochschule in Braunschweig $^{26}$. Vorausgegangen war eine deutsch-britische Historiker- und Geschichtslehrertagung im Juli 1950 ebenfalls in Braunschweig ${ }^{27}$, und erste Vorgespräche für die Institutsgründung hatte Eckert auf einem UNESCOSeminar im Juli/August desselben Jahres in Brüssel geführt, an dem 60 Delegierte $-»$ Erziehungsfachleute, Professoren, Lehrer, einzelne Verfasser und Herausgeber von Lehrbüchern« - aus 25 Ländern teilnahmen und sechs Wochen lang an der Verbesserung der Lehrbücher, speziell der Geschichtsbücher, arbeiteten ${ }^{28}$. Mit der Institutsgründung sollten die stürmisch einsetzenden, mannigfaltigen Initiativen und Projekte der Schulbuchrevision verstetigt, koordiniert und auch publiziert werden. So wurden die untersuchten Lehrbücher aus den beteiligten Ländern in Braunschweig von Anfang an systematisch gesammelt; sie bildeten den Grundstock für die gegenwärtig auf ca. 200000 Bände angewachsene, hinsichtlich der Provenienz ihrer Bestände einmalige Schulbuchbibliothek des Georg-Eckert-Instituts. Ebenso wichtig war die Entscheidung, die Aktivitäten der internationalen Schulbucharbeit einem breiteren Publikum, besonders Lehrem und Schulbuchverlagen, durch regelmäßige Veröffentlichungen zugänglich zu machen. Hierzu dienten das "Internationale Jahrbuch für Geschichtsunterricht«, dessen erster Band 1951 in Braunschweig erschien, und eine Schriftenreihe, in der neben größeren

26 21. bis 27. März 1951. Georg ECKERT, Die internationale Geschichtslehrertagung Ostern 1951, in: Internationales Jahrbuch für Geschichtsunterricht 1 (1951/52), S. 19lf. Wenig später wurde das Institut in "Internationales Schulbuchinstitut« umbenannt, eine Bezeichnung, die es bis zur Neugründung als "Georg-Eckert-Institut für internationale Schulbuchforschung « behielt.

${ }^{27}$ Die 2. deutsch-englische Geschichtslehrertagung in Braunschweig, in: Internationales Jahrbuch 1 (wie Anm. 26), S. 127-167; mit Beiträgen von Wilhelm MOMMSEN, Helmut KRAUSNICK, Charles H.C. BLOUNT, Otto-Ernst SCHÜDDEKOPF.

28 Das UNESCO-Seminar in Brüssel [...] vom 12.7. bis 23.8.1950, in: Internationales Jahrbuch 1 (wie Anm. 26), S. 180-190. 
Monographien hauptsächlich Tagungsmaterialien veröffentlicht worden $\operatorname{sind}^{29}$. Hier konnten sich die deutschen Leser über Wissensbestände im Ausland informieren, denn die Institutspublikationen enthielten auch essayartige Beiträge, wie beispielsweise Grundgedanken über den internationalen Ursprung einer Kultur von Lucien Febvre und François Crouzet $^{30}$. Sie kamen damit dem Bedürfnis entgegen, nach den Jahren der Abschottung durch den Nationalsozialismus wieder Anschluß an den internationalen geistigen Austausch zu finden und an ihm aktiv teilzuhaben. So schreibt Eckert über das schon erwähnte Brüsseler Seminar:

Wie bei vielen Konferenzen dürfte auch hier die persönliche Begegnung das Entscheidende und für die Zukunft Fruchtbringende gewesen sein. Das gilt natürlich in erhöhtem Maße für uns Deutsche. Nach den langen Jahren der Isolierung war es ein Erlebnis der besonderen Art, mit einem Kreis ausgesuchter Experten aus allen westlichen Ländern leben und arbeiten zu können, Erfahrungen auszutauschen, von Reformversuchen und Forschungen zu hören, die Kollegen in europäischen Nachbarländern, Amerika und Übersee in den letzten 10 bis 15 Jahren unternommen haben. Die wenigen freien Stunden wurden dem kostbaren Bücherschatz gewidmet, der fast überreichen Sammlung von Lehr- und Lernmitteln aus allen Ländern und Kontinenten, die die UNESCO zu diesem Zweck in Brüssel zusammengetragen hatte. Wir drei Deutschen, die mit großer Herzlichkeit in die Arbeits- und Lebensgemeinschaft des UNESCO-Seminars aufgenommen wurden, kamen jedenfalls ausgefullt mit Anregungen und neuen Eindrücken in die Heimat zurück. Wir hoffen, daß es uns gelingen wird, unsere Erfahrungen auch hier einem breiteren Kreis nutzbar zu machen. Diese Zeitschrift soll nicht zuletzt diesem Zweck dienen ${ }^{31}$.

Deutlicher kann man die Begeisterung, die Deutsche damals über die Teilnahme am internationalen Gedanken- und Erfahrungsaustausch empfanden, kaum ausdrücken.

Betreut wurden die Publikationen von dem Zeithistoriker Otto-Ernst Schüddekopf, Dozent an der Pädagogischen Hochschule Braunschweig, und lange Jahre der einzige Mitarbeiter Eckerts am Schulbuchinstitut. Beide bildeten ein Tandem, das sich ergänzte: Eckert war der nach außen wirkende Organisator und Mehrer, Schüddekopf der mehr im Innern tätige Sammler und Wahrer der Ernte ${ }^{32}$. Heute bilden diese frühen Institutspublikationen, deren relativ stetiges Erscheinen das Verdienst Schüddekopfs ist, eine wertvolle,

Ab Band 6 wurde der Titel geändert in »Internationales Jahrbuch für Geschichts- und Geographieunterricht«; bis $1977 / 78$ sind insgesamt 18 Bände erschienen; 1983 folgte ein Registerband. Abgelöst wurde das Jahrbuch durch die noch heute erscheinende Zeitschrift "Internationale Schulbuchforschung«. Die Schriftenreihe umfaßt mittlerweile über 100 Bände.

30 Lucien FEBVRE, François CROUZET, Der intemationale Ursprung einer Kultur. Grundgedanken zu einer Geschichte Frankreichs, in: Internationales Jahrbuch 2 (1953), S. 5-31.

31 Internationales Jahrbuch 1 (wie Anm. 26), S. 182.

32 Rainer RIEMENSCHNEIDER, Das Tandem Eckert - Schüddekopf und das Institutsgedächtnis, in: Internationale Verständigung (wie Anm. 3), S. 123. 
durch nichts $\mathrm{zu}$ ersetzende Quelle für die Rekonstruktion der Institutsgeschichte $^{33}$.

Umgekehrt waren die Schulbuchkonferenzen auch internationale Foren für die deutschen Historiker. Von Anfang an wurden auf ihnen u.a. brisante Themen der Zeitgeschichte, der jüngsten zumal, angesprochen. Dies wurde von den ausländischen Gesprächspartnern angemahnt, und Eckert wich diesen Forderungen nicht aus. So berichtete er über die schon erwähnte Gründungstagung des Schulbuchinstituts von Ostern 1951: »Den Höhepunkt bildete eine mit großer Offenheit und starker innerer Anteilnahme geführte Aussprache über das Dritte Reich und die deutsche Widerstandsbewegung, die, erst nach Mitternacht beendet, die ganzen folgenden Tage nachklang ${ }^{34}$. Eckert war aufgrund seiner Vita als Gegner des Nationalsozialismus ein sowohl qualifizierter wie auch bei ausländischen Kollegen hoch geachteter Gesprächspartner. Sein persönlicher Anteil an der mühseligen Wiederherstellung des deutschen Ansehens in der Welt und an der allmählich im Ausland einsetzenden Anerkennung der deutschen Bemühungen um eine "Bewältigung« der Vergangenheit kann vermutlich kaum überschätzt werden. Das Auswärtige Amt hat, wenn auch nicht aus ideologischer Übereinstimmung mit dem Denken Eckerts, so doch in realistischer Einschätzung seines wirkungsvollen Beitrags zur Völkerverständigung, die Arbeit des Schulbuchinstituts über Jahre hinweg finanziell zwar nicht besonders großzügig, aber doch zuverlässig unterstützt.

\section{Die deutsch-französischen Schulbuchkonferenzen}

Hatte sich das Netz der internationalen Schulbucharbeit des Instituts zunächst in der westlichen Welt bis nach Amerika und Japan erstreckt, so konnten in der Folge der neuen Ostpolitik der sozialliberalen Regierung Brandt/Scheel frühere, steckengebliebene Anläufe, mit Vertretern aus Ländern jenseits des Eisernen Vorhangs ins Gespräch zu kommen, wieder belebt werden. Damit setzte eine neue Phase in der Institutsarbeit ein. Doch bei aller Expansion der Aktivitäten weltweit ist die deutsch-französische Schulbucharbeit von Anfang an ein Grundpfeiler des Braunschweiger Instituts gewesen. Dies sei nachdrücklich betont, weil hierüber in der Forschung bisweilen unklare Vorstellungen bestehen. In einem anregenden Versuch einer Gesamtbilanz der deutsch-französischen Aussöhnungsbemühungen seit 1945 schreibt Hartmut Kaelble: »La Commission franco-allemande pour la révision des manuels scolaires d'histoire commença ses travaux à l'Institut international pour la recher-

33 Vgl. u.a. Otto-Ernst SCHÜDDEKOPF, Zwanzig Jahre westeuropäische Schulgeschichtsbuchrevision 1945-1965. Tatsachen und Probleme, Braunschweig 1966.

34 Die 2. deutsch-englische Geschichtslehrertagung in Braunschweig (wie Anm. 27), S. 191. 
che sur les manuels scolaires qui avait été fondé en Allemagne en $1975 \varkappa^{35}$. In diesem Satz sind gleich zwei Irrtümer enthalten. Zum einen ist das Internationale Schulbuchinstitut nicht erst 1975, sondern, wie gezeigt wurde, bereits 1951 gegründet worden. 1975, ein Jahr nach dem Tode Eckerts, erfolgte vielmehr seine Umwidmung zum Georg-Eckert-Institut für internationale Schulbuchforschung, was eine Statusänderung im Sinne der Schaffung eines selbständigen Forschungsinstituts, eine stärkere wissenschaftliche Fundierung der Institutsarbeit und einen bedeutenden Stellenausbau im wissenschaftlichen, bibliothekarischen und administrativen Bereich implizierte. Diese Neugründung durch den Gesetzgeber war im Grunde eine Anerkennung fuir ein Vierteljahrhundert intensiver Schulbucharbeit durch Georg Eckert. Indem Kaelble diese Vorgeschichte nicht erwähnt, nimmt er folgerichtig auch den Beitrag Eckerts zur Entwicklung der deutsch-französischen Schulbucharbeit nicht in den Blick. Er schreibt diese ausschließlich einer "groupe d'universitaires peu nombreux mais actifs « um Fritz Kern und Martin Göhring zu, die er - und dies zu Recht - als Motoren der frühen deutsch-französischen Verständigung bezeichnet ${ }^{36}$. Auch Georg Eckert darf zu diesem Personenkreis gezählt werden. Denn Eckert wurde gerade im deutsch-französischen Kontext sehr früh aktiv, mit der Konsequenz, die Winfried Schulze folgendermaßen definiert hat: Die deutsch-französischen Schulbuchkonferenzen nach 1950 haben »den Kern der Arbeit des Braunschweiger Schulbuchinstituts unter Georg Eckert« ausgemacht ${ }^{37}$.

Georg Eckert hat an der deutsch-französischen Historikertagung vom 9. bis 11. Oktober $1951 \mathrm{im}$ Institut für Europäische Geschichte in Mainz teilgenommen, auf der die »Deutsch-französische Vereinbarung über strittige Fragen europäischer Geschichte« verabschiedet wurde ${ }^{38}$. Vorbereitet wurde diese Tagung durch ein Treffen deutscher und französischer Historiker im Mai 1951 in Paris, zu dem Edouard Bruley, der Vorsitzende der Société des professeurs d'histoire et de géographie, eingeladen hatte, und das der Neubearbeitung der "Beschlüsse « von 1935 gewidmet war ${ }^{39}$. Diese beiden Veranstaltungen sind in personeller wie in historiographiegeschichtlicher Hinsicht bedeutsam. An den

Hartmut KAELBLE, La science et la réconciliation franco-allemande après 1945. Manuskript, französische Übersetzung von: Science and the Franco-German reconciliation since 1945, in: Technology and Society 23 (2001), S. 407-426, hier S. 413f.

${ }^{36}$ Ibid., S. 11. Vgl. auch die Beitrage von Heinz DuchHARDT und Corine DEFrancE in diesem Band.

37 Winfried SCHULzE, Deutsche Geschichtswissenschaft nach 1945, München 1989, S. 273.

38 Deutsch-französische Vereinbarung über strittige Fragen europäischer Geschichte, in: Internationales Jahrbuch 2 (1953), S. 78-109, zweisprachig.

39 Georg ECKERT, Die Revision der Thesen von 1935 auf der deutsch-französischen Historikertagung im Mai 1951, in: Internationales Jahrbuch 1 (wie Anm. 26), S. 65-67. Ein Nachdruck der "Thesen« von 1935: Ibid., S. 46-64, mit einer Einleitung von Jean Sigmann (S. 44-46). 
Teilnehmerlisten läßt sich gleichsam in Momentaufnahme und ausschnitthaft der damalige Stand des Integrationsprozesses der deutschen Historiker in den internationalen Kommunikationszusammenhang ablesen. Neben wichtigen Vertretern der Lehrerverbände waren an Wissenschaftlern beteiligt: Pierre Renouvin, Gaston Zeller, Jacques Droz, Jean-Baptiste Duroselle, Jean Sigmann, Edmond Vermeil; Georg Eckert, Karl Dietrich Erdmann, Martin Göhring, Hans Herzfeld, Helmut Krausnick, Gerhard Ritter, Helmut Röhr, Otto-Ernst Schüddekopf. Aus Zeitgründen oder wegen Visumschwierigkeiten verhindert waren Franz Schnabel und Gerd Tellenbach. Historiographiegeschichtlich sind die beiden Texte - die »Beschlüsse « von 1935 und die auf ihnen aufbauende und sie gleichzeitig erheblich modifizierende "Vereinbarung« von 1951 - aufschlußreich für den Grad der Konvergenzen bzw. der Divergenzen im deutsch-französischen Historikerdialog ${ }^{40}$. Die Divergenzen sind erheblich geschmolzen zugunsten der Konvergenzen, wie ein Vergleich der Texte von 1935 und 1951 ergibt. Rainer Bendick hat in einer eingehenden Untersuchung die Gründe für dieses Abschmelzen der Divergenzen dargelegt $^{41}$. Außerdem illustrieren die beiden Texte vorzüglich die Feststellung Kaelbles, daß der Rückgriff auf Präzedentien aus der Zwischenkriegszeit die Verständigung nach 1945 bisweilen erheblich erleichtert hat ${ }^{42}$. Eckert hat dafür gesorgt, daß die Beschlüsse von 1935, die in der deutschen Öffentlichkeit nahezu unbekannt geblieben waren ${ }^{43}$, im Jahrbuch veröffentlicht worden sind. Entsprechend auch die »Vereinbarung«, und zwar in deutscher und französischer Sprache. Die »Vereinbarung« hatte in der damaligen bundesdeutschen Öffentlichkeit viel Aufmerksamkeit gefunden; sie wurde in mehreren Organen, darunter auch der GWU, abgedruckt und ist in den folgenden Jahren immer wieder nachgedruckt worden, u.a. in hoher Auflage durch das Internationale Schulbuchinstitut im Jahre 1958 mit einem Vorwort von Hermann Heimpe ${ }^{44}$. Bundespräsident Theodor Heuss ging in einer Rede vor dem Bundestag am 14. Januar 1952 ausführlich auf sie ein: "Ich halte diesen scheinbar kleinen Vorgang für eine zentrale Leistung als Modell dessen, was möglich ist, um aus der propagandistisch hingenommenen und in den Verkrampfungen einer gestorbenen Aktualität verbliebenen Form des Geschichts-

40

41

42

43

44

Hierzu Rainer RIEMENSCHNEIDER, Verständigung und Verstehen. Ein halbes Jahrhundert deutsch-französischer Schulbuchgespräche, in: Hans-Jürgen PANDEL (Hg.), Verstehen und Verständigen, Pfaffenweiler 1991, S. 137-148.

Rainer BENDICK, Irrwege und Wege aus der Feindschaft. Deutsch-französische Schulbuchgespräche im 20. Jahrhundert, in: Kurt HochSTUHL (Hg.), Deutsche und Franzosen im zusammenwachsenden Europa 1945-2000, Stuttgart 2003, S. 73-103.

KAELBLE, La science (wie Anm. 35), S. 11.

SCHULZE, Deutsche Geschichtswissenschaft (wie Anm. 37), S. 273.

Deutsch-französische Vereinbarung über strittige Fragen europäischer Geschichte. Neudruck März 1958, Braunschweig 1958. 
bildes herauszukommen ${ }^{45}$. Diese Erklärung war sowohl an die deutsche wie an die französische Öffentlichkeit gerichtet. Sie unterstrich den Beitrag, den die Wissenschaft zur Versachlichung von emotional stark aufgeladenen Streitpunkten leisten kann. Die deutsch-französische »Vereinbarung« hat eine ähnlich starke Öffentlichkeitswirkung erzielt wie zwanzig Jahre später die deutsch-polnischen Schulbuchempfehlungen, denen die "Vereinbarung« sowohl hinsichtlich des Procedere als auch der Zwecksetzung als Modell diente $^{46}$. Sie hat also »stilbildend" gewirkt, um einen Ausdruck Karl-Dietrich Erdmanns aufzunehmen.

Von Historikern erarbeitet, an die Lehrer gerichtet, das war auf eine kurze Formel gebracht das Wesen der internationalen Schulbuchrevision. Eckert war stets bemüht, für die Konferenzen die besten Fachkollegen von den Universitäten und Forschungsinstituten zur Mitarbeit zu gewinnen, um die wissenschaftliche Qualität der Arbeitsergebnisse zu sichern. Das ist ihm in vielen Fällen auch gelungen. Manche machten aus der Überzeugung mit, daß es sich dabei um eine wichtige Angelegenheit handelte. Außerdem ergab sich die Gelegenheit, für die eigene Arbeit Kontakte zu knüpfen, was Eckert als durchaus legitim empfand und auch begrüßte. Andere wiederum waren wegen der Aussicht dabei, auf Staatskosten eine Auslandsreise machen zu können, was in der Nachkriegszeit ein besonders verlockendes Motiv war. Manche Vertreter der Fachwissenschaft jedoch haben die Mitarbeit abgelehnt - entweder aus politischen Gründen oder weil sie der Ansicht waren, daß sie aus der Beschäftigung mit Schulbüchern denn doch herausgewachsen seien.

Was immer die Motive für eine Mitarbeit bei den internationalen Schulbuchgesprächen gewesen sein mochten - fest steht, daß diese Mitarbeit unter den politischen und psychologischen Bedingungen der Nachkriegszeit nicht ohne Risiko war. Kaelble erinnert in seiner Untersuchung daran, daß die Mitstreiter des Mainzer Instituts als "Reichsfeinde ${ }^{47}$ apostrophiert wurden. Georg Eckert erging es nicht anders. Die Pressedossiers, die zu der Arbeit des Internationalen Schulbuchinstituts zusammengestellt worden sind, enthalten nicht nur positive, zustimmende Reaktionen, und Eckert war häufig der Adressat unverhohlener Droh- und Schmähbriefe. Noch zwanzig Jahre später wurden die deutsch-polnischen Empfehlungen dadurch diskreditiert, daß sie ähnlich wie die "Ostpolitik« der Regierung Brandt/Scheel - nach Meinung ihrer Gegner einen wohlfeilen Ausverkauf wohlverstandener deutscher

45 Zitiert in: Internationales Jahrbuch 2 (1953), S. 109, im Anschluß an den Abdruck der Vereinbarung.

46 Rainer RIEMENSCHNEIDER, Transnationale Konfliktbearbeitung. Das Beispiel der deutsch-französischen und der deutsch-polnischen Schulbuchgespräche im Vergleich, 1935-1998, in: Carsten TESSMER (Hg.), Das Willy-Brandt-Bild in Deutschland und Polen, Berlin 2000, S. 121-131.

47 KAELBLE, La science (wie Anm. 35), S. 11. 
Interessen darstellten ${ }^{48}$. Im Verständnis derer jedoch, die den Frieden auf der Grundlage des Verzichts auf Gewaltanwendung nicht nur deklamierten, sondern auch praktizierten, hat westdeutsche Politik nichts preisgegeben, /was die Väter [nicht bereits] verloren haben ${ }^{49}$. Die Rückkehr der deutschen Geschichtswissenschaft in die »Ökumene der Historiker« führte eben über einen steinigen Weg.

\section{Anhang}

\section{Beiträge zum Geschichtsunterricht}

BARION, Jakob, Macht und Recht und das Wesen des Staates, Braunschweig 1951.

CzYBULKA, Gerhard, Die Lage der ländlichen Klassen Ostdeutschlands im 18. Jahrhundert, Braunschweig 1949.

Deutsche Außenpolitik 1933-1945. Dokumente mit verbindendem Text, Braunschweig ${ }^{6} 1963$.

DITTMER, Kunz, Vom Grabstock zum Pflug. Ein Gang durch die Wirtschaftsgeschichte, Braunschweig 1949.

ECKERT, Georg, Arbeiterleben in der Frühzeit des Industriekapitalismus, Braunschweig 1949.

-, Aus den Lebensberichten deutscher Fabrikarbeiter, Braunschweig 1954.

-, Der Bauernkrieg, Braunschweig 1948.

-, Der Freiherr vom Stein und die preußischen Reformen, Braunschweig 1948.

-, Das junge Deutschland und die Revolutionsdichtung des Vormärz, Braunschweig 1948.

-, Der Merkantilismus, Braunschweig 1949.

-, Quellen zur Wirtschafts- und Sozialgeschichte des 17./18. Jahrhunderts, Braunschweig 1949.

-, Die Revolution von 1848/49, Braunschweig 1947.

-, Vom Bismarckreich zur Republik, Braunschweig 1949.

MIELCKE, Karl (Hg.), Bismarck, Otto von, Briefe, Berichte, Denkschriften, Erlasse, Gespräche, Reden, Verträge, Braunschweig 1954.

- (Hg.), Dokumente zur Geschichte der Weimarer Republik, Braunschweig 1956.

48 Siehe hierzu die Dokumentation von JACOBMEYER, Die deutsch-polnischen Schulbuchempfehlungen (wie Anm. 2).

49 Willy BRANDT im Bericht zur Lage der Nation zu Jahresbeginn 1970, Erinnerungen. Mit einem aktuellen Vorwort, Frankfurt a.M. 1992, S. 216. 
- (Hg.), Innenpolitik 1933-1945. Das nationalsozialistische Herrschaftssystem. Dokumente mit verbindendem Text, Braunschweig ${ }^{5} 1961$.

-, Bismarck in der neueren Forschung, Braunschweig 1954.

-, Das deutsche Bürgertum und die Reichsgründung, Braunschweig 1948.

-, Die Französische Revolution, Braunschweig [ca. 1949].

-, Urkunden und Berichte zur Geschichte der Französischen Revolution, Braunschweig [ca. 1949].

-, Geschichte der Weimarer Republik, Braunschweig 1951.

-, Städtewesen und Frühkapitalismus, Braunschweig 1948.

-, Der Vormärz. Vom Abschluß des Wiener Kongresses 1815 bis zum Ausbruch der Revolution im März 1848, Braunschweig 1949.

-, Das Zeitalter der Entdeckungen, Braunschweig 1948.

PETRY, Ludwig, Die Gegenreformation in Deutschland, Braunschweig 1952.

REYNOLDS, Philip A., Die britische Außenpolitik zwischen den beiden Weltkriegen, Braunschweig 1952.

RODENSTEIN, Heinrich, Die Utopisten, Braunschweig 1949.

SCHÜDDEKOPF, Otto-Ernst, Die deutsche Innenpolitik im letzten Jahrhundert und der konservative Gedanke. Die Zusammenhänge zwischen Außenpolitik, innerer Staatsführung und Parteiengeschichte, dargestellt an der Geschichte der Konservativen Partei von 1807 bis 1918, Braunschweig 1951.

FREDE, Günther, Otto-Ernst Schüddekopf, Wehrmacht und Politik 1933-1945. Dokumente mit verbindendem Text, Braunschweig ${ }^{4} 1963$.

SPIES, Otto, Orientalische Kultureinflüsse im Abendland, Braunschweig 1949. TREuE, Wilhelm, Wirtschaft und Politik 1933-1945. Dokumente mit verbindendem Text, Braunschweig ${ }^{4} 1964$.

TRIMBORN, Hermann, Das Menschliche ist gleich im Urgrund aller Kulturen, Braunschweig 1948.

WENZEL, Fritz, Der junge Luther, Braunschweig 1948. 
